\title{
Revisión crítica sobre el síndrome del latigazo cervical (I): ¿de veras existe una lesión anatómica?.
}

\author{
A critical review of whiplash associated disorders (I): \\ Is there really an anatomical lesion?.
}

\section{A. Ortega Pérez 1}

\begin{abstract}
RESUMEN
El síndrome del latigazo cervical (SLC) tiene una incidencia aproximada de I caso/ 1000 personas y año y unos costes personales y económicos muy altos. El mecanismo lesional puede ser más o menos complejo, según las direcciones relativas de los vehículos y la posición de la cabeza en el momento del impacto. Su clave parecen ser unos movimientos no fisiológicos de las vértebras cervicales y tan rápidos que los músculos que normalmente los frenan no tienen tiempo de hacerlo. En los impactos puede lesionarse cualquier estructura del cuello, aunque lo más frecuente es la afectación de las vértebras $\mathrm{C} 5$ y $\mathrm{C} 6$ y la lesión del cartílago o de la cápsula de las articulaciones interapofisarias y la de los ligamentos vertebrales comunes anterior o posterior. Sin embargo, las radiografías, las TAC y las RM son pruebas poco apropiadas para detectar esas lesiones, sutiles pero clínicamente relevantes y la regla en el SLC es la falta de pruebas iconográficas de lesión cervical. Las radiografías simples suelen hallar lesiones degenerativas previas o la rectificación de la lordosis cervical, un signo inespecífico de lesión. La RM es más sensible, pero tiene un cierto porcentaje de falsos positivos, con detección de lesiones sin correlación clínica. La falta de imágenes de lesión no debe llevar al médico forense a pensar que no hay lesiones.
\end{abstract}

Palabras clave: Diagnóstico, incidencia, lesiones anatómicas, mecanismo de la lesión, radiografía, resonancia magnética, revisión, síndrome del latigazo cervical.

\section{ABSTRACT}

Whiplash associated disorders (WAD) have an approximate incidence of I case/I 000 persons per year, and with high personal and economic costs. The mechanism of lesion may be more or less complex, depending on the relative directions of the colliding vehicles and on the orientation of the head at the moment of impact. In any case, the key seems to be the non-physiologic movements of the cervical vertebrae which are so rapid that the muscles normally controlling them do not have enough time to perform their function. On impact, virtually any part of the neck can be damaged, although the most frequent lesions affect the zygapophyseal joint facet, capsules or cartilages of either the fifth or sixth cervical vertebrae, as well as the anterior or posterior longitudinal ligaments. However, standard radiographies, CT scans or MRI are not appropriate explorations for the detection of these subtle, but clinically relevant lesions and the norm in WAD is the absence of image evidence of cervical lesion. Standard radiography generally detects previous degenerative changes or a cervical kyphotic angle, a non-specific sign of lesion. MRI is more sensitive, but has a high percentage of false positives, i.e., it detects lesions that do not have any clinical relevance.

Key words: Anatomic lesions, diagnostic, incidence, lesion mechanism, magnetic resonance imaging, radiography, review, whiplash associated disorders.

Fecha de recepción: 22.AGO.03

Fecha de aceptación: 23.MAR.03

Correspondencia: Dr. Arturo Ortega. Unidad de Medicina Legal. Facultad de Medicina y Ciencias de la Salud. C/ Sant Llorenç, 21. 43201 - Reus (Tarragona). Correo electrónico: aop@fmcs.urv.es.

1 Doctor en medicina. Profesor de medicina legal y toxicología. Facultad de Medicina y Ciencias de la Salud. Universidad "Rovira i Virgili". 


\section{INTRODUCCIÓN:}

El "síndrome del latigazo cervical" (SLC) es una de las lesiones con las que los médicos forenses se enfrentan con más frecuencia, pero demasiado a menudo les desorienta y les despierta aprensión. Los motivos de ese sentimiento los resume bien un artículo reciente "La evolución del dolor es imprevisible. La causa es desconocida porque no se ha objetivado ninguna lesión. El problema médico se puede complicar cuando hay reivindicaciones laborales o petición de incapacidad." [I]. Lo cierto es que las dos primeras frases pueden explicar dos errores frecuentes entre los médicos clínicos (incluso entre algunos traumatólogos) y en los médicos forenses. De ahí la necesidad de revisar las características clave del síndrome y de criticar las asunciones que pueden sesgar la labor de estos.

Los altísimos costes personales y económicos del síndrome del latigazo cervical y su gran incidencia convierten a esta lesión en uno de los campos clave de la tarea del médico forense. Este facultativo deberá valorar los daños somáticos y psíquicos padecidos por los afectados, los cuidados médicos que necesiten, la duración de la incapacidad laboral transitoria y, si el lesionado tiene secuelas, cómo le afectan en su ocupación y en sus actividades habituales, por ejemplo, menoscabando su productividad laboral o incluso impidiéndole trabajar.

El hecho de que las manifestaciones del SLC, así como su duración, sean tan variadas, unido a la ausencia de alteraciones en las pruebas de imagen, no debe ser la excusa para atribuir a la ligera el síndrome a trastornos psíquicos del paciente o a la simulación con el fin de conseguir una compensación económica.

Creo poder demostrar en este artículo que la lesión anatómica existe, pero que difícilmente es apreciable con las pruebas de imagen a nuestro alcance en medicina clínica. Las distintas lesiones anatómicas son las responsables de la variedad de las manifestaciones y de su duración.

El trastorno tiene cerca de cien años de historia, ya que los primeros casos, descritos como "columna de los viajeros del tren", ocurrieron a personas implicadas en accidentes ferroviarios en los inicios del siglo $X \times[2]$.

El grupo de trabajo de Québec sobre trastornos asociados al latigazo cervical lo define como una transferencia de energía al cuello resultado de una aceleración-desaceleración, que puede provenir de un impacto desde atrás o desde un lado, predominantemente por accidentes de vehículos a motor. La energía transferida puede causar lesiones óseas o de los tejidos blandos, las cuales a su vez pueden llevar a una amplia variedad de manifestaciones clínicas.

\section{INCIDENCIA DEL SÍNDROME DEL LATIGAZO CERVICAL:}

Aunque la incidencia exacta de este trastorno es desconocida, podemos estimarla en I caso por cada mil personas y año. Sin duda aumenta paulatinamente en todo el mundo.

Los datos que justifican esa incidencia son los siguientes: Cassidy et al. [3] estudiaron los casos en una región de Canadá. En un año y medio recogieron datos de 15.738 pacientes para un área con I, I millones de habitantes. La tasa de afectados sería de 0,95 casos/ / 000 habitantes y año. En un estudio prospectivo danés [4] reclutaron a las personas que habían sufrido un latigazo cervical si cumplían una serie de criterios de inclusión. 232 personas los cumplían en un período de reclutamiento de un año y en un área con 284.000 habitantes. Eso daría 0,8 casos/I000 personas y año, teniendo en cuenta que no todos los afectados están incluidos. En otro estudio prospectivo, quebequés [5], para una población total de 135.000 personas registraron 446 lesionados que habían sufrido un latigazo cervical y habían acudido al servicio hospitalario de urgencias a lo largo de dos 
años y medio. La incidencia anual sería en este caso de I,3 casos/I 000 personas y año, aunque de nuevo es una estimación conservadora.

En cambio, Sterner et al. [6] citan una incidencia anual mucho mayor, de 4,2 casos por mil habitantes, tal vez debida a la abundancia de vehículos y a la frecuencia de uso de estos.

\section{EL MECANISMO DE LA LESIÓN:}

Los estudios biomecánicos nos han obligado a desechar la explicación clásica de 'latigazo' en favor de una nueva hipótesis, más compleja. Además, en condiciones reales, las diferentes posiciones en las tres dimensiones del espacio en las que pueden estar la cabeza y el cuello de los viajeros nos muestran la limitación de los modelos teóricos.

La explicación clásica del mecanismo de la lesión le dio el nombre de "latigazo cervical", tan gráfico: el impacto empujaba hacia delante al vehículo alcanzado, y con él al tronco y los hombros del ocupante. Como la cabeza y el cuello estaban menos sujetos, por inercia aquella quedaba atrás un instante, provocando la hiperextensión del cuello. Pero inmediatamente después se adelantaban con rapidez, hasta la hiperflexión. Estos movimientos rápidos y forzados serían los responsables de las lesiones articulares y de los tejidos blandos.

Sin embargo, los estudios biomecánicos con videocámaras de alta velocidad, acelerómetros, cinerradiografía [7] y electromiografía [8] han permitido obtener más detalles sobre los movimientos de la cabeza y del cuello después de una colisión, desembocando en una nueva hipótesis sobre los movimientos vertebrales en las primeras centésimas de segundo después del impacto: la primera respuesta del cuello, antes de que se desplacen las vértebras cervicales superiores y la cabeza, es el movimiento hacia atrás de la vértebra C6, hasta alcanzar su extensión máxima. Cuando la alcanza, fuerza a la vértebra C5 a extenderse. Es decir, que en esos primeros momentos las vértebras cervicales inferiores estarían extendidas mientras que las superiores estarían en una flexión relativa, dando a la columna cervical en conjunto una forma de S, en lugar de su C habitual (Figura I). Después, el movimiento del tronco hacia delante continúa y la cabeza "se queda atrás", con lo que se extiende toda la columna cervical. A partir de aquí sigue el movimiento clásico de latigazo. Esa secuencia de movimientos anómala sería la causante de las lesiones por latigazo cervical $[7,8,9]$.

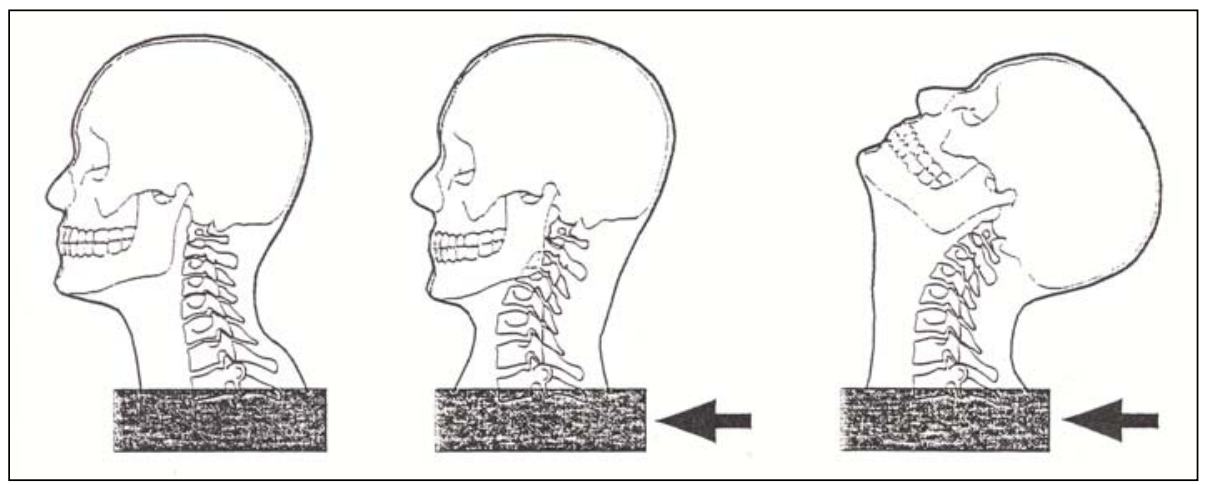

Figura 1.- Representación del movimiento de la columna cervical después de un impacto desde atrás (tomada de [8]). Izquierda: posición neutra del cuello antes del impacto. Centro: en los primeros 60 milisegundos después del impacto, el tórax y las últimas vértebras cervicales se desplazan hacia delante, con lo que la columna cervical adopta una forma de S. Derecha, unos 100 milisegundos después del impacto, toda la columna cervical está extendida. A partir de aquí continuaría el movimiento clásico de latigazo. 
El modelo descrito explica la situación más sencilla, con un choque exactamente desde atrás y mientras la cabeza del individuo afectado está en posición anatómica, es decir, horizontal y mirando hacia delante. En esta situación, todos los movimientos ocurren en el plano sagital. Pero por supuesto en la realidad en el momento del impacto el individuo puede tener la cabeza flexionada ventral o dorsalmente, inclinada hacia un lado o rotada y los choques entre los vehículos pueden ser frontales, laterales u oblicuos. En definitiva, en los impactos reales los movimientos de la cabeza son más complejos y tienen consecuencias más lesivas para la columna cervical del afectado que los del modelo clásico.

No ocurrre así con un choque frontal, que provoca una aceleración de la cabeza 2,8 veces menor que la causada por un golpe desde atrás y con una sucesión de movimientos más sencilla que en este [10]. En cambio, cuando la cabeza está rotada, las tensiones sobre las vértebras al flexionar y extender la columna son mayores que si está en posición anatómica [I I]. Pero es que el impacto hacia delante sobre una cabeza rotada aumentará la rotación antes de que se produzca la extensión vertebral (por las posiciones relativas del centro de gravedad de la cabeza y del eje del cuello), lo que agravará la situación. Esa rotación de la columna cervical hace más susceptibles a la lesión a las articulaciones interapofisarias, a los discos intervertebrales y a los ligamentos [12].

En definitiva, el cuello se mueve de formas no fisiológicas y los músculos que normalmente ayudan a regular la dirección y la amplitud de los movimientos no tienen tiempo de responder a las fuerzas aplicadas sobre este [13].

La columna cervical puede lesionarse incluso en choques a poca velocidad: del 75 al $90 \%$ de las lesiones cervicales ocurren a velocidades inferiores a $25 \mathrm{~km} / \mathrm{hora}[14,15,16]$ o algo más altas: una mediana de $40 \mathrm{~km} / \mathrm{hora}$, con el $50 \%$ central entre 30 y $55 \mathrm{~km} / \mathrm{hora}$ [4]. Como es de esperar, la mayoría de los autores encuentran una relación directa entre la violencia del impacto y el dolor tras la lesión [17].

\section{¿EXISTE UNA LESIÓN ANATÓMICA EN EL SLC?:}

Los afectados sufren lesiones en los músculos, los ligamentos, las cápsulas articulares, los discos o los nervios, pero pocas veces se fracturan o luxan las vértebras cervicales, salvo que el choque haya sido muy violento. Por eso las pruebas de imagen comunes tienen poco rendimiento diagnóstico y, dado el informe de 'normalidad', cuesta aceptar que existen lesiones en el cuello. Sin embargo, dichas lesiones se han demostrado por otros métodos.

Los españoles Combalía et al. [12] estudiaron registros de observaciones clínicas, de estudios con cadáveres y de pruebas complementarias y citan una lista impresionante de lesiones anatómicas que pueden ocurrir a consecuencia de un latigazo cervical "las articulaciones interapofisarias (rotura de la cápsula articular, efusión hemática, fisuras, lesiones del cartílago articular), discos intervertebrales (desinserción, fisuración y rotura del annulus), músculos (roturas parciales o totales con hematomas), ligamentos (rotura del ligamento vertebral común anterior, el interespinoso, el vertebral común posterior y el amarillo), región atlas-axis (fractura de odontoides, entre otras; lesiones ligamentosas), vértebras cervicales (fracturas desapercibidas), cerebro (hematomas y hemorragias), otras estructuras (articulación temporomandibular, nervio simpático cervical, avulsión del occipital por arrancamiento del ligamento nucal, parálisis de las cuerdas vocales, lesión de la médula espinal sin fracturas etc.)." Muchas de estas son difícilmente detectables o directamente invisibles en las pruebas de imagen (Figura 2). 
En una revisión previa, Suso y Mateos [13] daban más detalles sobre las pruebas que apoyan la existencia de esas lesiones.

Yoganandan et al. [18] apreciaron dichas lesiones anatómicas disecando los tejidos. Situaron cadáveres como pasajeros de automóviles, que después fueron sometidos a alcances a velocidades de tan solo 16 ó 25 km/hora. Tras la colisión congelaron los cadáveres y los seccionaron con un criomicrotomo. En la parte inferior de la columna cervical hallaron: distensión y desgarro del ligamentum flavum, rotura del annulus, ruptura del ligamento vertebral común anterior y alteración de la articulación interapofisaria con desgarro de los ligamentos capsulares (Figura 3). La mayoría de lesiones se situaban entre las vértebras C5 y C6, lo esperable de la extensión de la porción inferior de la columna al inicio del movimiento de latigazo.

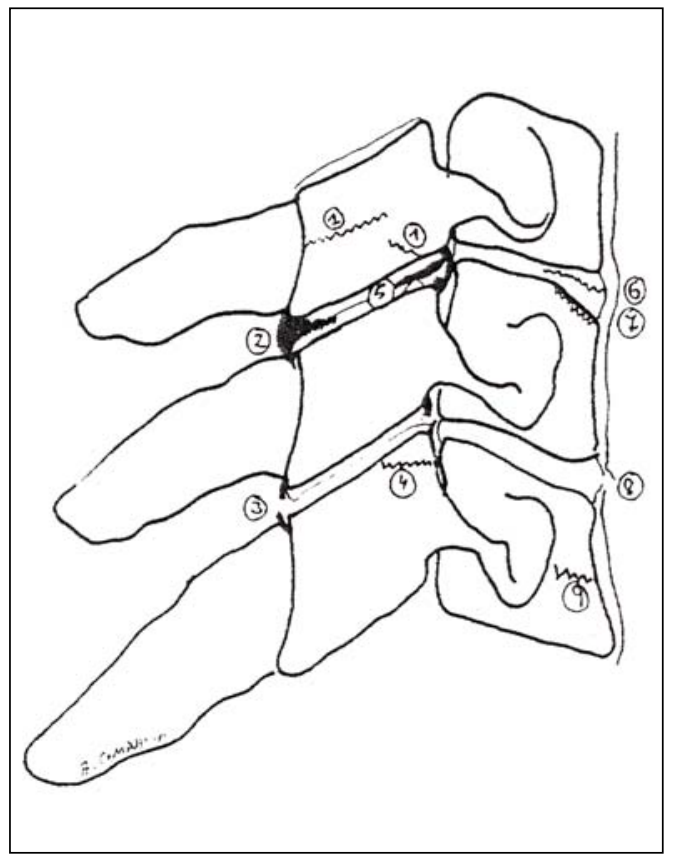

Figura 2.- Posibles lesiones de la columna cervical tras un latigazo cervical: 1) pequeños aplastamientos trabeculares de las apófisis articulares; 2) efusión hemática de las articulaciones interapofisarias; 3) desgarro y rotura de la cápsula y ligamentos de las articulaciones interapofisarias; 4) fracturas del ápex de la apófisis articular; 5) fracturas del cartílago y desgarros sinoviales; 6) fisuraciones del disco intervertebral; 7) fractura-acuñamiento mínima del cuerpo vertebral; 8) desgarros del ligamento vertebral común anterior, y 9) roturas trabeculares del cuerpo vertebral. Todas ellas son difíciles de identificar o directamente invisibles en las pruebas de imagen. Tomada de [12].

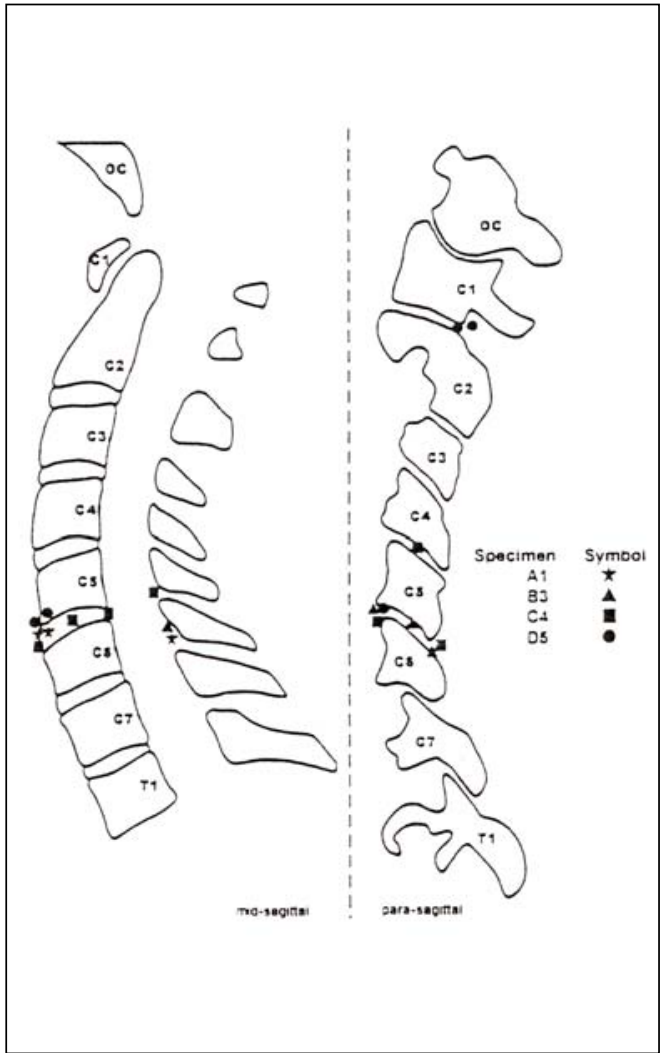

Figura 3.- Esquema de la localización de las lesiones en los individuos estudiados por Yoganandan et al. [18]. A la derecha, lesiones en las articulaciones interapofisarias vistas en la sección parasagital. A la izquierda, otras lesiones vistas en la sección sagital media. Tomada de [18].

Los datos disponibles son aún más concretos: las articulaciones interapofisarias son la víctima más común de los movimientos combinados de cizallamiento, flexión y compresión [19], especialmente si la cabeza estaba rotada hacia un lado en el momento del impacto. Y los estudios clínicos lo han confirmado: en una investigación doblemente a ciegas y controlada mediante placebo, 
Lord et al. demostraron que las carillas articulares de las articulaciones interapofisarias vertebrales eran la fuente de dolor en el cuello en el 60 \% de los afectados por dolor crónico tras sufrir un síndrome de latigazo cervical [20].

Combalía et al. resumen en su revisión [12] el llamado modelo de lesión en el latigazo cervical, basado en que el traumatismo puede afectar a estructuras diferentes. La mayoría de los traumatismos -que son los más leves- causarán un esguince o un desgarro muscular. Como el músculo tiene capacidad de recuperación, la lesión mejorará, desapareciendo en unas semanas. Sin embargo, algunas sacudidas lesionarán los discos, las articulaciones interapofisarias o los ligamentos, o combinaciones de estos, que no curarán, sino que se convertirán en una fuente de dolor crónico.

\section{PRUEBAS DE IMAGEN PARA REFRENDAR EL DIAGNÓSTICO:}

Si existen lesiones anatómicas en el SLC ipor qué no las detectamos con las radiografías, las tomografías axiales computadas (TAC) o las resonancias magnéticas (RM)? ¿Por qué la regla es la falta de pruebas iconográficas de lesión cervical? La explicación, por chocante que pueda parecer, es que no son las pruebas adecuadas para detectar estas lesiones. Podemos ilustrar las frases anteriores con lo ocurrido a Ruiz et al. [2।], médicos forenses que trabajaron con |3| pacientes diagnosticados de SLC. La radiografía simple fue normal en 96 de estos casos y en los otros 35 encontraron signos degenerativos. Realizaron 13 RM a sendos pacientes, ocho de las cuales fueron normales, mientras que en las otras cinco aparecieron hernias discales de significado clínico dudoso.

\section{Radiografías simples:}

En la gran mayoría de estos pacientes las radiografías se interpretan como normales para el grupo de edad del afectado. Entre las alteraciones, la más común son los cambios degenerativos preexistentes, aunque puede encontrarse un ligero aplanamiento de la lordosis cervical normal, indicador de la contractura muscular causada por el dolor [22].

Ronnen et al. [23] estudiaron a 100 pacientes con SLC y antes de que transcurrieran tres semanas les practicaron radiografías de la columna cervical, incluyendo tomas funcionales. En las radiografías de 17 pacientes aparecía una cifosis segmentaria, pero en la RM posterior no apreciaron lesiones de los tejidos blandos. Los autores concluyeron que la cifosis segmentaria muy probablemente era atribuible a la hipermovilidad de la columna por encima de las vértebras bloqueadas por el espasmo muscular.

Subirana et al., médicos forenses, publicaron un caso clínico de inestabilidad ligamentosa postraumática entre las vértebras C4 y C5, que había sido pasado por alto al realizarse solo radiografías cervicales anteroposterior y lateral [24].

No solo es difícil el diagnóstico de las lesiones de partes blandas, sino también el de determinadas fracturas óseas. Con las radiografías cervicales simples anteroposterior y lateral pasan desapercibidas la mayoría de las fracturas de los macizos articulares o de las articulaciones interapofisarias [13].

\section{Resonancia magnética:}

La TAC y la RM son técnicas sofisticadas, pero tampoco con ellas pueden identificarse las lesiones de partes blandas en la fase aguda $[3,25,26]$. 
Borochgrevink et al. [27] practicaron una RM del cerebro y del cuello a pacientes en los dos primeros días después del latigazo cervical, pero no hallaron diferencias entre lesionados y controles.

Ronnen et al. [23] practicaron RM de la columna cervical y del cerebro a 100 pacientes. sólo en uno de ellos encontraron una lesión relacionada con el accidente, un edema prevertebral. No detectaron las lesiones de partes blandas que sugerían las cifosis segmentarias en las radiografías simples. La conclusión de los autores fue que era innecesario practicar RM a los pacientes con radiografías normales y sin manifestaciones de déficit neurológico.

Van Geothem et al. [28] aconsejaban reservar la TAC y la RM para los lesionados con déficit neurológico, sospecha de lesión discal o medular, fractura o lesión ligamentosa. Y los hallazgos de Pettersson et al. [29] apoyaban esa recomendación. Estos autores practicaron una exploración clínica y una RM a 39 individuos que habían sufrido pocos días antes un latigazo cervical. Hallaron lesiones discales en 25 de ellos (hernias discales en 10 de los lesionados) y una lesión muscular en el otro. Veintidós de los 26 lesionados con RM patológicas tenían signos neurológicos, lo mismo que les ocurría a 7 de los 10 individuos con hernia discal.

En una investigación algo posterior [30] hallaron hernias discales en 13 de los 39 lesionados con SLC. Los autores concluyeron que la RM en la fase aguda del SLC tenía demasiados falsos positivos, lo que dificultaba la correlación de sus hallazgos con las manifestaciones clínicas iniciales. La RM estaría indicada más adelante, en los pacientes con manifestaciones persistentes (dolor en la extremidad superior, déficit neurológicos o signos de compresión de la raíz nerviosa), con el fin de diagnosticar una hernia discal que debiera ser intervenida quirúrgicamente.

Una investigación ya citada [18] confirma la escasa sensibilidad de las pruebas de imagen. De los cuatro cadáveres sometidos a un choque, y que tenían lesiones anatómicas, solo habían encontrado anomalías radiográficas (y eran anodinas), en dos. En las TAC también hallaron alteraciones solo en dos sujetos, aunque en uno la lesión era importante, una distracción de la articulación interapofisaria derecha entre C5 y C6.

Para mejorar la correlación entre las quejas del enfermo y las imágenes de lesión o incluso los trastornos durante el funcionamiento de la columna, necesitaremos nuevas proyecciones con las técnicas actuales o nuevas técnicas de imagen, aún más sofisticadas. Van Geothem et al. [28] proponían las tomas en flexión o extensión, la RM estática de alta resolución y en particular la RM dinámica. Pero mientras tanto, la falta de imágenes de lesión no debe inducir a la equivocación de menospreciar los síntomas del paciente.

En la tabla I están resumidas las conclusiones prácticas de la primera parte de esta revisión. En la segunda parte abordaré otros apsectos del SLC, entre ellos su diagnóstico clínico, la influencia de las compensaciones económicas en la duración de los trastornos, los estudios sobre su duración y los factores pronósticos de su persistencia. 
- La incidencia aproximada del SLC es de 1/1000 personas y año. Aumentará paulatinamente en todo el mundo.

- El mecanismo clásico de 'latigazo' ha sido desplazado por una nueva hipótesis, más compleja. Aún así, no explica lo que ocurre cuando la cabeza y el cuello están flexionados o rotados.

- La columna cervical puede lesionarse incluso en choques a $25 \mathrm{~km} / \mathrm{hora}$ o menos.

- Las radiografias, las TAC y las RM suelen informarse como 'normales', porque pocas veces se fracturan o luxan las vértebras cervicales. Las lesiones están en los músculos, los ligamentos, las cápsulas articulares, los discos o los nervios.

- La mayoría de lesiones se sitúan entre las vértebras C5 y C6.

- Las carillas articulares de las articulaciones interapofisarias son la fuente de dolor en el $60 \%$ de los afectados por dolor crónico en el cuello tras sufrir un SLC.

- El 'modelo de lesión en el latigazo cervical' propone que la mayoria de los traumatismos causarán un esguince o un desgarro muscular, lesión que mejorará y desaparecerá en unas semanas. Sin embargo, algunas sacudidas lesionarán los discos, las articulaciones interapofisarias o los ligamentos, o combinaciones de estos, que sin poder curar, se convertirán en una fuente de dolor crónico.

- La gran mayoria de las radiografías del cuello de estos pacientes se interpretan como normales para el grupo de edad del afectado. Como mucho, se aprecian cambios degenerativos, preexistentes, o un ligero aplanamiento de la lordosis normal, indicador de la contractura muscular causada por el dolor, con hipermovilidad de la columna por encima de las vértebras bloqueadas por ese espasmo.

- Con las radiografias cervicales simples anteroposterior y lateral pasan desapercibidas la mayoría de las fracturas de los macizos articulares o de las articulaciones interapofisarias.

- La correlación entre las manifestaciones del lesionado y los hallazgos de la TAC y la RM sólo es significativa en aquellos con déficit neurológico o manifestaciones persistentes, que lleven a la sospecha de lesión discal o medular, fractura o lesión ligamentosa.

- La ausencia de imágenes de lesión no debe llevar a minusvalorar las manifestaciones del lesionado.

Tabla 1.- Deducciones prácticas de esta primera parte de la revisión. 


\section{BIBLIOGRAFÍA:}

I. Roig D. Patología cervical (I): Síndromes cervicales sin afección neurológica. Jano 2001; 60:63-64.

2. Crowe $H$. Injuries to the cervical spine. Presented at the annual meeting of the Western Orthopaedic Association, San Francisco, California, 1928.

3. Cassidy JD, Carroll LJ, Côté P et al. Effect of eliminating compensation for pain and suffering on the outcome of insurance claims for whiplash injury. N Engl J Med 2000;342:I179-1186.

4. Kasch H, Stengaard-Pedersen K, Arendt-Nielsen L, Jensen TS. Headache, Neck Pain, and Neck Mobility After Acute Whiplash Injury. Spine 2001;26:1246-125I.

5. Hartling L, Brison RJ, Ardern C, Pickett W. Prognostic Value of the Quebec Classification of Whiplashassociated Disorders. Spine 200I;26:36-4I.

6. Sterner Y, Toolanen G, Gerdle B, Hildingsson C. The incidence of whiplash trauma and the effects of different factors on recovery. J Spinal Disord Tech 2003; 16:195-199.

7. Kaneoka K, Ono K, Inami S, Hayashi K. Motion analysis of cervical vertebrae during whiplash loading. Spine 1999;24:763-769.

8. Cusick JF, Pintar FA, Yoganandan N. Whiplash syndrome. Kinematic Factors Influencing Pain Patterns. Spine 2001;26:12521258.

9. Grauer JN, Panjabi MM, Cholewicki J, et al. Whiplash produces an S-shaped curvature of the neck with hyperextension at lower levels. Spine 1997;22: 2489-2494.

10. Croft AC, Haneline MT, Freeman MD. Low speed frontal crashes and low speed rear crashes: is there a differential risk for injury? Annu Proc Assoc Adv Automot Med 2002; 46:79-91.

II. Winkelstein BA, Nightingale RW, Richardson W], et al. The cervical facetcapsule and its role in whiplash injury: a biomechanical investigation. Spine 2000;25: I238-1246.

12. Combalía A, Suso S, Segur JM, García S, Alemany FX. Síndrome del latigazo cervical. Med Integral 2001;38:95-102.

13. Suso S, Mateos $G$. Síndrome del latigazo cervical. Ponencia presentada en las Terceras jornadas catalanas de actualización en medicina forense. Barcelona, noviembre de 1995. Editado por la Generalitat de Catalunya.

14. Eichberger A, Darok M, Steffan H, Leinzinger PE, Bostrom O, Svensson MY. Pressure measurements in the spinal canal of post-mortem human subjects during rearend impact and correlation of results to the neck injury criterion. Accid Anal Prev 2000;32:251-260.

15. Eichberger A, Geigl BC, Moser A et al. Comparison of different car seats regarding headneck kinematics of volunteers during rear end impact. Proceedings of the International Research Council on Biomechanics of Impact Conference, Dublin, Ireland, 1996:153-164.
16. Hell W, Langwieder K. Reported soft tissue neck injuries after rearend car collisions. International Research Council on Biomechanics of Impact Conference, Goteborg, Sweden, 1998:261-274.

17. Obelienne D, Schrader H, Bovim G, et al. Pain after whiplash: A prospective controlled inception cohort study. J Neurol Neurosurg Psychiatry 1999;66:279-283.

18. Yoganandan N, Cusick JF, Pintar FA,Rao RD. Whiplash injury determination with conventional spine imaging and cryomicrotomy. Spine 2001;26:2443-2448.

19. Siegmund GP, Myers BS, Davis MB, Bohnet HF, Winkelstein BA. Mechanical Evidence of Cervical Facet Capsule Injury During Whiplash. Spine 2001;26: 2095-2101.

20. Lord SM, Barnsley L, Wallis B] et al. Chronic cervical zygapohyseal joint pain after whiplash: a placebocontrolled prevalence study. Spine 1996;21:1737-1745.

2I. Ruiz JL, Guerado E, Godino M, De los Santos MI. Abordaje terapéutico del síndrome del latigazo cervical agudo. Rev $S$ And Traum y Ort 2001;21:23-27.

22. Smith E, Mayans J, Montañana JV et al. Esguince cervical postraumático. Valoración lesional y terapéutica. Repercusiones médicas y legales. Rev $S$ And Traum y Ort 2001;21:189-194.

23. Ronnen HR, de Korte PJ, Brink PR, van der Bijl HJ, Tonino AJ, Franke CL Acute whiplash injury: is there a role for MR imaging?a prospective study of 100 patients. Radiology 1996;201:93-96.

24. Subirana M. Cuquerella A, Reig R. iSíndrome del latigazo cervical con secuelas 0 error diagnóstico inicial? En: Libro de las Cuartas jornadas catalanas de actualización en medicina forense. Barcelona, noviembre de 1997. Editado por la Generalitat de Catalunya.

25. Scholten-Peeters GGM, Bekkering GE, Verhagen AP, et al. Clinical Practice Guideline for the Physiotherapy of Patients With Whiplash-Associated Disorders. Spine 2002;27:412-422.

26. Hildingsson C, Hietala SO, Toolanen G. Scintigraphic findings in acute whiplash injury of the cervical spine. Injury 1989;20:265-266.

27. Borochgrevink G, Smevik 0, Haave I, et al. MRI of cerebrum and cervical column within two days after whiplash neck sprain injury. Injury 1997;28:331-335.

28. Van Geothem JW, Biltjes IG, van den Hauwe L, Parizel PM, De Schepper AM. Whiplash injuries: is there a role for imaging? Eur J Radiol 1996;22:30-37.

29. Pettersson K, Karrholm J, Toolanen G, Hildingsson C. Decreased width of the spinal canal in patients with chronic symptoms after whiplash injury. Spine 1995; 20:1664-1667.

30. Pettersson K, Hildingsson C, Toolanen G, Fagerlund M, Bjornebrink J. Disc pathology after whiplash injury. A prospective magnetic resonance imaging and clinical investigation. Spine 1997;22:283-287. 